\title{
Integral indicators of the swimming techniques effectiveness of highly qualified crawl-stroke swimmers
}

\author{
Krylov A.I. ${ }^{1 \mathrm{ABCDE}}$, Gorelov A.A. ${ }^{2 \mathrm{ABCDE}}$, Tretyakov A.A. ${ }^{3 \mathrm{ABCDE}}$ \\ ${ }^{1}$ Lesgaft National State University of Physical Education, Sport and Health, Russia \\ ${ }^{2}$ Saint Petersburg University of the Ministry of Internal Affairs of the Russian Federation, Russia \\ ${ }^{3}$ Belgorod law Institute of the Ministry of internal affairs of Russia named after I.D. Putilin, Russia
}

Authors' Contribution: A - Study design; B - Data collection; C - Statistical analysis; D - Manuscript Preparation; E- Funds Collection.

\begin{tabular}{|c|c|}
\hline \multicolumn{2}{|l|}{ Abstract } \\
\hline Purpose: & $\begin{array}{l}\text { It is known that prominent world-class swimmers are characterized by the manifestation of specific } \\
\text { abilities. These abilities allow to constantly maintain a stable speed swimming of different segments of } \\
\text { the distance. In this case, highly qualified swimmers can increase the maximum speed of swimming on } \\
\text { a segment of a given length. They achieve this by increasing the total external mechanical power. In this } \\
\text { case, swimmers keep, and in some cases even reduce the power of the stroke. It is implemented the } \\
\text { phase-specific principle of creating driving forces during each phase. At the same time, the principle } \\
\text { of creating driving forces is common to all swimming locomotion. The article gives a rationale for the } \\
\text { effectiveness of the integral criterion application for evaluating swimming techniques. The integral } \\
\text { criterion for evaluating the swimming technique was applied at remote speeds of the entire spectrum of } \\
\text { the competitive front crawl swimming program. }\end{array}$ \\
\hline Material: & $\begin{array}{l}\text { The study involved } 9 \text { highly qualified crawl-stroke swimmers, members of Russia national team. The age } \\
\text { of the participants ranged from } 18 \text { to } 24 \text { years. It is studied the kinematic and kinetic characteristics of the } \\
\text { front crawl swimming technique of highly qualified athletes at various competitive distances. } \\
\text { The studies applied video recording of swimmer's movements with OLYMPUS TG- } 5 \text { camera (Vietnam) } \\
\text { from a depth of } 4.5 \text { m. Three luminous markers located on the swimmer's hips were applied to record } \\
\text { the dynamic parameters of swimmer's movements. The results of the swims were processed with } \\
\text { Natatometry }{ }^{\mathrm{TM}} \text { (Russia). }\end{array}$ \\
\hline Results: & $\begin{array}{l}\text { It was determined that swimmers demonstrated high indices of the intra-cyclic dynamic index (ICDI - } \\
\text { Intra Cycle Dynamic Index) and the dynamic coordination index of the swimming cycle (DCI - Dynamic } \\
\text { Coordination Index) at speeds in medium distances relative to speeds at sprint distances. } \\
\text { Swimmers demonstrate the ability to generate a significant amount of promotional efforts at } 100 \mathrm{~m} \\
\text { distance that ensure the achievement of high speeds. It is established that no general trends in ICDI } \\
\text { changes in individual structural phases of the stroke. This indicates the demonstration of individual } \\
\text { specific features in the swimming technique of each athlete. }\end{array}$ \\
\hline Conclusions: & $\begin{array}{l}\text { It is considered the feasibility of applying the integral indicators ICDI and DCI for a quantitative assessment } \\
\text { of the intra cycle promotion forces interaction and hydrodynamic resistance forces arising at the level of } \\
\text { an integral biomechanical system of the swimming cycle. }\end{array}$ \\
\hline Keyw & wimmers, high qualification, front crawl, performance indices, swimming technique. \\
\hline
\end{tabular}

\section{Introduction}

The modern crawl swimming competition program is very diverse. Swimmers cover a distance in a wide range of speeds. The change in speed applies different requirements to the athlete's body. These requirements should be specific to power supply mechanisms. Should affect the biomechanical characteristics of swimming technique [1].

For highly qualified world-class swimmers, the manifestation of specific abilities is characteristic only for a specific individual. [2]. Such abilities allow you to constantly maintain a stable swimming speed on the different segments of the distance [3]. In this case, highly qualified swimmers can increase the maximum speed of swimming on a segment of a given length. This is due to the increase in total external mechanical power [4]. Power of the stroke, in this case, may be maintained or even reduced. This phenomenon indicates the so-called hidden

\footnotetext{
C) Krylov A.I., Gorelov A.A., Tretyakov A.A., 2019

doi:10.15561/18189172.2019.0402
}

possibilities of the individual [1]. These features can be realized in reaching the maximum swimming speed (CDP-phenomenon). The active hydrodynamic resistance of the swimmer is reduced in order to do that [5].

The basis of the propulsion mechanism of the stroke in swimming was determined in another study [6]. The basis is the strength of the hand cross. It includes the cumulative effect of hydrodynamic lifting force and frontal resistance (in the middle phases); hydrodynamic lifting force (at the end of the stroke). Hydrodynamic resistance increases due to the working movements of the athlete. The value of this increase in rowing is from 15 to $22 \%$, and in swimming - up to $100 \%$ [7]. This characterizes the manifestation of individual technical skill.

This position has been confirmed in other studies [8, 9]. It stipulates further scientific research. The search should be aimed at developing an integral criterion for evaluating the effectiveness of swimming techniques. Such efficiency is determined at the remote speeds of the competitive front crawl swimming program. The 
importance of solving this problem is manifested in evaluating the effectiveness of training exercises. The developed criteria allow to receive urgent information about the effectiveness of swimming techniques; make corrections to the training process.

In modern swimming, there is intense competition in every type of program. As a result, all-round swimmers appear. Such athletes show high results in the sprint and long distance. This is especially characteristic of the style "front crawl".

Studies of Kravcov [4] deal mainly with the kinematic characteristics of the swimming technique. Studies show that at submaximal speeds, highly qualified athletes achieve increased speed by unifying the technique's quality. Unification does not imply an increase in the power stroke and an increase in energy consumption [10]. Improvement of technology occurs due to the optimization of the tempo-rhythm structure of the stroke.

Therefore, it is necessary to supplement the kinematic analysis of the swimming cycle with experimental dynamic characteristics $[11,12,13]$.

Hypothesis. The emergence of CDP-phenomenon is stipulated by the ability of athletes to select the biomechanical characteristics of the technique in accordance with the objectives for a particular competitive distance.

The purpose of the study is to analyze the abilities of "all-around crawl swimmers" to select the biomechanical characteristics of the technique in accordance with the objectives of a particular competitive distance.

\section{Material and methods}

Participants. The study involved highly qualified crawl swimmers $(n=9)$, members of Russia national Team. The age of athletes ranged from 18 to 24 years. The height of athletes ranged from $180 \mathrm{~cm}\left(5^{\prime} 11\right.$ ") to
$191 \mathrm{~cm}$ (6’3”), weight - from $73 \mathrm{~kg}$ (160 pounds) to $82 \mathrm{~kg}$ (181 pounds). All athletes were informed of the purpose, objectives of the experiment and research procedures.

Design of the study.

Studies were conducted in a 25-meter indoor swimming pool of the Lesgaft National State University of Physical Education, Sport and Health.

All participants in the experiment were offered to crawl three $25 \mathrm{~m}$ distances. The distance competitive speed of each athlete is the same as in swimming 100, 200 and $1500 \mathrm{~m}$. Each athlete performed the exercises individually with the choice of speed rate that is applied at rating competitions.

The studies applied video recording of swimmer's movements with OLYMPUS TG-5 camera (Vietnam) from a depth of $4.5 \mathrm{~m}$. (Fig. 1). Three luminous markers located on the swimmer's hips were applied to record the dynamic parameters of swimmer's movements.

The following tasks were solved In the process of special research:

1. Development of an experimental method for determining the basic dynamic characteristics of front crawl swimming. It was studied the swimming cycle in athletes with various speed swimming techniques.

2 . The study of swimming speed changes patterns and the dynamic structure of the swimming cycle.

For processing the results of the swims, the Natatometry ${ }^{\mathrm{TM}}$ program developed by us was applied [14]. The following kinematic and dynamic characteristics of swimming technique were obtained:

1. The average speed in the swimming cycle $-\mathrm{v}$.

2. The distance covered by a swimmer in one swimming cycle $-\mathrm{d}$.

3 . Time of one swimming cycle $-t$.

4. The absolute amount of swimmer accelerations in a cycle - a.

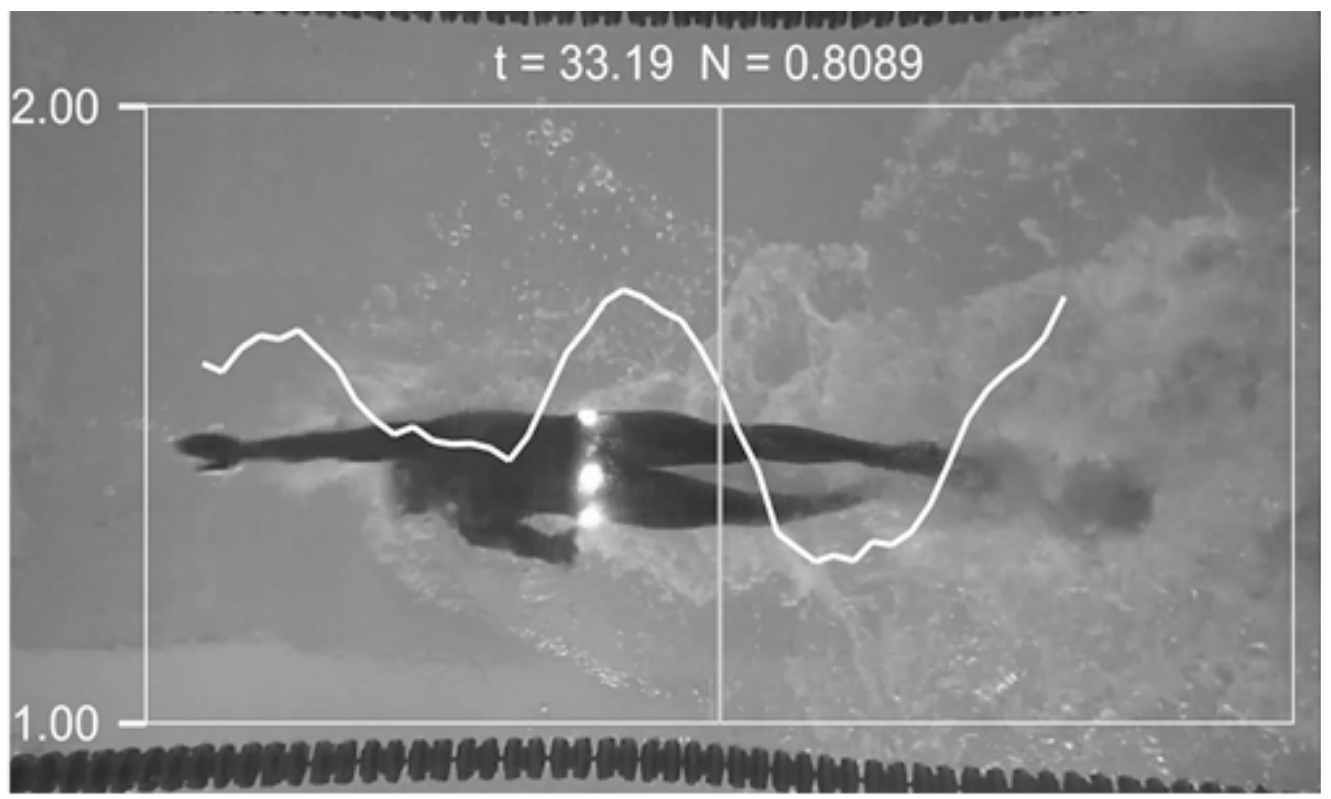

Fig. 1. An example of a shot of an underwater video recording with an overlaid graph of intra cycle speed. The ordinate axis is time, the abscissa is instantaneous speed $(\mathrm{m} / \mathrm{s})$. The vertical line in the center of the graph indicates the point on the graph that corresponds to this shot. 
These parameters were also calculated for each dynamic phase of the stroke: the propulsive phase, the phase of slowdown and the phase of active slowdown [7].

In crawl, swimming athlete performs hand strokes in turn. Calculations were carried out for each hand separately.

To solve the research problems, the following integral indicators were calculated:

Intra Cycle Dynamic Index (ICDI)

$$
\mathrm{ICDI}=\frac{\mathrm{DVavg}}{|\mathrm{a}|} \times 1000
$$

$\mathrm{D}$ - the length of the swimming cycle (m);

Vavg - average speed in the cycle $(\mathrm{m} / \mathrm{s})$;

$|\mathrm{a}|$ - the sum of absolute values in the cycle.

2. The sum of the intra cycle dynamic indices $(\Sigma)$ of each dynamic phase of the swimming cycle. For a crawl -6 phases, 3 phases with the right hand, 3 phases with the left hand:

$\Sigma=\Sigma(\mathrm{r})+(\mathrm{l})$

where,

$\Sigma(\mathrm{r})$ - sum of right hand dynamic phases ICDI;

$\Sigma(1)$ - sum of left hand dynamic phases ICDI.

1. Dynamic Coordination Index of the swimming cycle (DCI - Dynamic Coordination Index).

Dynamic Coordination Index (DCI):

$$
\mathrm{DCI}=\frac{\mathrm{ICDI}}{\sum}
$$

The measurement results are presented in table 1.

For the convenience of data analysis, Vavg speeds were divided into three ranges;

1) Average $(1.4 \div 1.6 \mathrm{~m} / \mathrm{s})$ - speeds at a distance of $1500 \mathrm{~m}$ crawl;

2) Submaximal $(1.7 \div 1.8 \mathrm{~m} / \mathrm{s})$ - speeds at a distance of $200 \mathrm{~m}$ crawl;

3) High $(1.9 \mathrm{~m} / \mathrm{s}$ and above $)$ - speeds at a distance of $100 \mathrm{~m}$ crawl.

The results of the swims were processed by Natatometry ${ }^{\mathrm{TM}}$ program [14]. The kinematic and dynamic characteristics of the swimming technique were calculated by Natatometry ${ }^{\mathrm{TM}}$ program.

The following integral indices were calculated:

1. Intra Cycle Dynamic Index (Intra Cycle Dynamic Index - ICDI);

2. The sum of the intra cycle dynamic indices $(\Sigma)$ of each dynamic phase of the swimming cycle. For a crawl - 6 phases, 3 phases with the right hand, 3 phases with the left hand;

3. Dynamic Coordination Index of the Swimming Cycle (DCI -Dynamic Coordination Index) Dynamic Coordination Index (DCI).

Statistical analysis.

The intra cycle dynamic index, the sum of the intra cycle dynamic indices (the sum of right and left hands) and the dynamic coordination index of the swimming cycle were obtained during the study. These characteristics were calculated using the SPPS.20 program.

The results of the swims were processed by Natatometry ${ }^{\mathrm{TM}}$ (program Russia).

It was conducted correlation analysis of the swimming speed and the intra cycle dynamic index, the swimming speed and the dynamic coordination index for a more indepth study.

\section{Results}

Integral indicators should be developed based on the interrelated biomechanical and hydrodynamic characteristics of the swimming cycle. Therefore, the position was formulated in the course of applying new research methods with modern technology and computer technique. Such equipment can operate in the aquatic environment.

The correlation diagram of the average swimming speed Vavg and the Intra Cycle Dynamic Index (ICDI) is presented in Figure 2. The correlation diagram of Vavg and Dynamic Coordination Index (DCI) is presented in Figure 3.

The average speed in the swimming cycle (Vavg) ranged from 1.3 to $2,1 \mathrm{~m} / \mathrm{s}$.

For the convenience of data analysis, we have divided Vavg speeds into three ranges:

1) Average $(1.4 \div 1.6 \mathrm{~m} / \mathrm{s})-$ speeds at a $1500 \mathrm{~m}$ distance crawl;

2) Submaximal $(1.7 \div 1.8 \mathrm{~m} / \mathrm{s})-$ speeds at a $200 \mathrm{~m}$ distance;

3) High (1.9 m / s and above) - speeds at $100 \mathrm{~m}$ distance crawl.

It was performed the analysis of the diagram. All selected athletes showed high ICDI and DCI indices at medium distances' speeds, relative to sprint distances' speeds.

This demonstrates the ability of all-around swimmers to reduce significantly the energy expenditure at the stayer distances.

At the same time, they demonstrate the ability to generate a significant amount of promotional effort. These efforts allow you to reach high speeds at a $100 \mathrm{~m}$ distance.

It should be noted that in the course of research we did not establish general trends in ICDI changes in individual structural phases of the stroke. This indicates the manifestation of individual specific features in the swimming technique of each athlete.

Some swimmers achieve a speed increase by increasing the power of the stroke or the correct distribution of physical effort during the second phase (propulsion). Other swimmers achieve the same results by reducing the forces of hydrodynamic resistance in the 1st slip phase and the 3 rd phase of active resistance.

All athletes were characterized by very high indices of integral coefficients when swimming at submaximal speeds corresponding to $200 \mathrm{~m}$ distances $(1.7 \div 1.8 \mathrm{~m} / \mathrm{s})$.

Swimming at such speeds requires the maximum mobilization of anaerobic glycolytic abilities and is accompanied by heavy feelings of fatigue. 
Table 1. Integral characteristics of the swimming cycle of crawl-stroke swimmers participated in the research

\begin{tabular}{|c|c|c|c|c|c|c|c|c|c|c|c|c|}
\hline \multirow{2}{*}{ № } & \multirow{2}{*}{ Swim } & \multicolumn{4}{|c|}{ left hand phase ICDI } & \multicolumn{4}{|c|}{ right hand phase ICDI } & \multirow{2}{*}{$\boldsymbol{\Sigma}$} & \multirow{2}{*}{ ICDI } & \multirow{2}{*}{$\begin{array}{l}\mathrm{DCl} \\
(\%)\end{array}$} \\
\hline & & Iph & IIph & IIIph & $\Sigma I$ & Iph & IIph & IIIph & $\Sigma r$ & & & \\
\hline 1 & pp_100 & 40.3 & 87.1 & 131.1 & 258.5 & 36.6 & 99.3 & 55.7 & 191.6 & 450.1 & 210.1 & 0.46 \\
\hline 2 & pp_200 & 24.3 & 67.4 & 83.4 & 175.1 & 60.4 & 132.2 & 30.8 & 223.4 & 398.5 & 311.3 & 0.78 \\
\hline 3 & pp_1500 & 45.7 & 99.3 & 59 & 204 & 40.3 & 118.5 & 21.4 & 180.2 & 384.2 & 250.4 & 0.65 \\
\hline 4 & kl_100 & 24 & 133.4 & 59.5 & 216.9 & 65.8 & 78.8 & 43.5 & 188.1 & 405 & 191.3 & 0.47 \\
\hline 5 & kl_200 & 82.4 & 59 & 40.1 & 181.5 & 75.4 & 195.1 & 0 & 270.5 & 452 & 367.1 & 0.81 \\
\hline 6 & kl_1500 & 46.1 & 60.9 & 82.3 & 189.3 & 58.5 & 89.9 & 95.1 & 243.5 & 432.8 & 241.9 & 0.6 \\
\hline 7 & sv_100 & 60.4 & 120.2 & 70.2 & 250.8 & 57.7 & 59.3 & 33.4 & 150.4 & 401.2 & 212.9 & 0.47 \\
\hline 8 & sv_200 & 58.8 & 123.5 & 35.4 & 217.7 & 62.4 & 204.2 & 0 & 266.6 & 484.3 & 401.8 & 0.82 \\
\hline 9 & sv_1500 & 45.2 & 45.5 & 40.9 & 131.6 & 80 & 120.4 & 90.5 & 290.9 & 422.5 & 321.5 & 0.76 \\
\hline 10 & iv_100 & 38.9 & 88.7 & 29 & 156.6 & 31.8 & 67.1 & 10.8 & 109.7 & 266.3 & 177.3 & 0.66 \\
\hline 11 & iv_200 & 79.9 & 67.7 & 68.2 & 215.8 & 77.1 & 203.2 & 0 & 280.3 & 496.1 & 398.8 & 0.80 \\
\hline 12 & iv_1500 & 85.9 & 88.3 & 91.4 & 265.6 & 54.1 & 86.4 & 26.1 & 166.6 & 432.2 & 300.9 & 0.69 \\
\hline 13 & pr_100 & 55.4 & 10.5 & 28.4 & 94.3 & 54.6 & 90.8 & 35.4 & 180.8 & 275.1 & 159.4 & 0.57 \\
\hline 14 & pr_200 & 81.2 & 110.2 & 40.4 & 231.8 & 60.1 & 112.2 & 29.4 & 201.7 & 433.5 & 321.7 & 0.74 \\
\hline 15 & pr_1500 & 50.8 & 51.2 & 28.4 & 130.4 & 39.1 & 135 & 46.2 & 220.3 & 350.7 & 209.6 & 0.59 \\
\hline 16 & sp_100 & 61.4 & 87.1 & 38.3 & 186.8 & 28.7 & 70.4 & 12.2 & 111.3 & 298.1 & 130.8 & 0.43 \\
\hline 17 & sp_200 & 106.2 & 73.4 & 100.2 & 279.8 & 45.8 & 109.9 & 66.5 & 222.2 & 502 & 420.2 & 0.83 \\
\hline 18 & sp_1500 & 57.8 & 65.1 & 63.2 & 186.1 & 61.7 & 89.3 & 50.4 & 201.4 & 387.5 & 288.1 & 0.74 \\
\hline 19 & ba_100 & 19.4 & 40.7 & 88.6 & 148.7 & 35.2 & 58.4 & 18.7 & 112.3 & 261 & 157.7 & 0.60 \\
\hline 20 & ba_200 & 77.5 & 95.4 & 61.3 & 234.2 & 52.2 & 81.4 & 20.9 & 154.5 & 388.7 & 304.3 & 0.78 \\
\hline 21 & ba_1500 & 53.9 & 55.6 & 45.9 & 155.4 & 45.3 & 71.7 & 33.1 & 150.1 & 305.5 & 206.6 & 0.67 \\
\hline 22 & ka_100 & 30.4 & 60.4 & 11.2 & 102 & 27.1 & 60.9 & 10.1 & 98.1 & 200.1 & 109.3 & 0.54 \\
\hline 23 & ka_200 & 56.1 & 88.4 & 78.3 & 222.8 & 73.2 & 110.1 & 14.8 & 198.1 & 420.9 & 350.9 & 0.83 \\
\hline 24 & ka_1500 & 82.1 & 68.3 & 28.2 & 178.6 & 49.9 & 68.1 & 10.3 & 128.3 & 306.9 & 228.3 & 0.74 \\
\hline 25 & db_100 & 40.3 & 33.2 & 22.4 & 95.9 & 35.1 & 54.2 & 12 & 101.3 & 197.2 & 100.8 & 0.51 \\
\hline 26 & db_200 & 98.7 & 51.1 & 33.2 & 183 & 36.3 & 125 & 64.8 & 226.1 & 409.1 & 360.1 & 0.88 \\
\hline 27 & $\mathrm{db} \_1500$ & 28.7 & 60.2 & 10.9 & 99.8 & 76 & 80.6 & 31.9 & 188.5 & 288.3 & 177.4 & 0.61 \\
\hline
\end{tabular}

Note: pp_100 - certain athlete swimming a 100 m distance; left hand phase ICDI - intra cycle dynamic index in the left hand's stroke phases; right hand phase ICDI - intra cycle dynamic index in the right hand's stroke phases; Iph, IIph, IIIph $-1,2,3$ stroke's phases; $\Sigma l, \Sigma r$ - the sum of the indices in the stroke's phases of the right and left hands; - the sum of the indices of the right and left hands; ICDI - intra cycle dynamic index; DCI - dynamic coordination index.

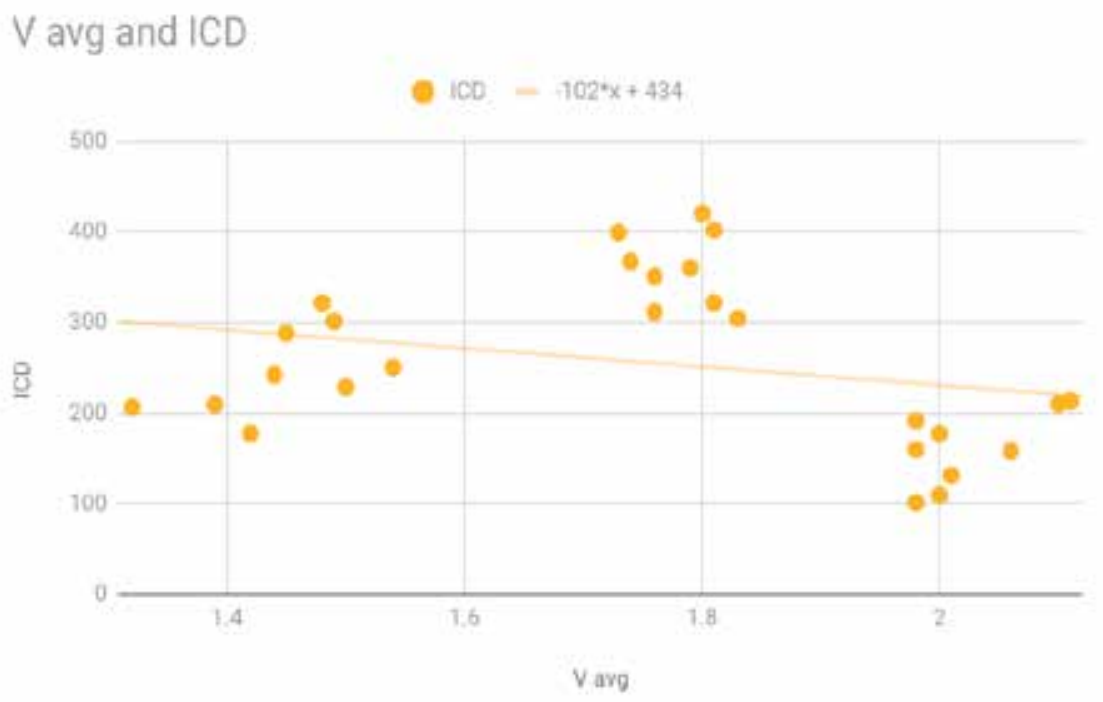

Fig. 2. Correlation diagram of Vavg (average swimming speed) and ICDI (intra cycle dynamic index). 
Table 2. Correlation analysis of the integral characteristics of the swim cycle of crawl-stroke swimmers

\begin{tabular}{llll}
\hline & Vavg & ICDI & DCI \\
\hline Vavg & 1 & $-0,3616$ & $-0,4623$ \\
ICDI & $-0,3616$ & 1 & 0,85808 \\
DCI & $-0,4623$ & 0,85808 & 1 \\
\hline
\end{tabular}

Note: Vavg - the average speed in the cycle; ICDI - intra cycle dynamic index; DCI - dynamic coordination index.

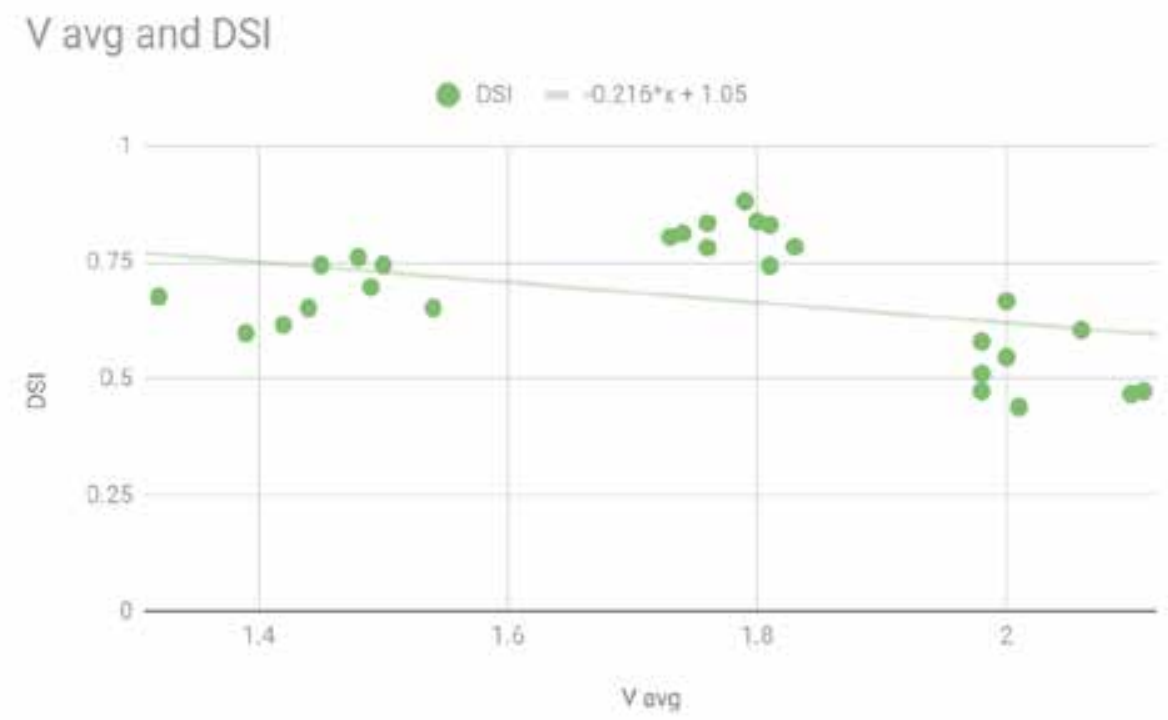

Fig. 3. Vavg and $\mathrm{DCl}$ Correlation diagram.

To achieve high levels of endurance, swimmers must have a very flexible, variable technique. This technique allows to achieve a specified level of swimming speed. At the same time, there are significant correlations in the dynamic and spatial-temporal characteristics of the motor actions.

It was determined that highly qualified swimmers achieve an increase in speed by increasing the power of the propulsive efforts while simultaneously reducing the forces of hydrodynamic resistance.

So, for three swimmers in swims No. 5, No. 8 and No. 11 , the active braking phase is practically absent, that is, the propulsive phase continues until the end of the stroke.

\section{Discussion}

Issurin [6] conducted a metrological analysis of the swimmer motor actions' kinematics. This approach was applied in developing the method of recording and analyzing the intra cycle speed. It is based on computer processing of swimmer promotion parameters. It was performed with an underwater video camera. The widespread use of photographic registration in sports research determines the number of advantages of applying this method. The advantages of this method include: the autonomy of the object of study; the possibility of conducting research at some distance from the object; the possibility of simultaneous analysis of the kinematic parameters and the relative position of the segments of the athlete's body; selection of kinematic characteristics along the coordinate axes [14].

In our studies, the video registration of the swimming technique allows to determine the kinematic characteristics of the swimmer's motor actions in each specific shot of the video. It gives the opportunity to assess the effectiveness of changes or correlations in the swimming intra cycle speed. The developed technique greatly expands the possibilities of photo registration and film cyclography. The technique allows to assess the effectiveness of the implementation of training exercises [9. 14]. The developed criteria provide an opportunity to receive urgent information about the effectiveness of swimming techniques and, accordingly, to make corrections into the training process [7].

Our study of the swimmer's translational motion kinematics provides an opportunity to analyze the main sources of error [11]. It also makes it possible to formulate general requirements for the implementation of training exercises and for the technique of a particular athlete.

It was defined that the kinematic phase structure of the stroke in swimming can only be considered in the complex with its dynamic characteristics. This allows to improve significantly the efficiency of swimming techniques in general.

Other studies argued [1, 3, 15-18] the need to determine the swimmer's speed as a constant value. Although it is not so. The magnitude of the speed 
correlations greatly affects the efficiency of swimming techniques. Any random speed deviation during a single swimming cycle is a recognized fact [19]. Therefore, it is necessary to determine the indicators of propulsive efficiency, considering the non-stationary translational you to make a video of the swimmers' motor actions by camera "OLYMPUS TG-5" (Vietnam) from a depth of 4.5 $\mathrm{m}$. You must use the three luminous markers located on the swimmer's hips.

The study allowed: to expand the capabilities of the swimming techniques analysis; consider different swimming speed and power. The developed intra cyclic dynamic index (ICDI) and dynamic coordination index (DCI) have shown their effectiveness in assessing the kinematic characteristics of swimming techniques. The presented indices allowed to analyze the common factors of changes in the swimming speed and the dynamic structure of the swimming cycle. The study also allowed to identify the third dynamic phase of the stroke in the front crawl, as a phase of active slowdown. In this phase of the stroke, the speed of the rowing hand rises, and the motion of the swimmer's body in the water. This allows

intra cycle speed begins to decrease. These studies were not conducted in the studied literature.

\section{Conclusions}

Integral indicators of the effectiveness' evaluation of swimming technique (ICDI - intra cycle dynamic index; DCI - dynamic coordination index of the swimming cycle) allows to quantify the interaction of intra cycle propulsive forces and hydrodynamic resistance forces. They arise at the level of a complete biomechanical system of the swimming cycle. These data confirm the results of our experimental studies.

The use of these indices makes it possible to compare the dynamic characteristics with key kinematic (intra cycle indicators of speed and acceleration) at competitive and training speeds. This will help improve the quality of technical training for highly qualified swimmers.

\section{Conflict of interest}

The authors declare that there is no conflict of interest regarding the publication of this article.

macrocycle training. Fizicheskaia kul'tura, sport - nauka $i$ praktika, 2018;4:23-30. (In Russian)

1. Kolmogorov S, Rumyantseva O, Gordon B, Cappaert J. Hydrodynamic characterristics of competitive swimmers of different genders and performance levels. Journal of Appllied Biomechanics. 1997; 13: 20- 17. https://doi.org/10.1123/jab.13.1.88

2. Gorelov AA, Noskov MS, Tret'iakov AA, Drogomereckij VV. Individualization as a basis for increasing the effectiveness of sports activities. Modern problems of science and education np. 2015;1: 20-30. (In Russian)

3. Mosunov DF. Assessment of kinetic energy of system "swimmer-water" in movement cycle. Uchenye zapiski universiteta imeni P.F. Lesgafta, 2017;4: 146-152. (In Russian)

4. Kravcov AM. Method of urgent control and correction of swimming technique in competitions and training exercises. Moscow: TVT Division, 2010. (In Russian)

5. Mosunov DF. The method of application analysis of intracyclic speed in swimming. Adaptivnaia fizicheskaia kul'tura, 2013;4:56-64. (In Russian)

6. Issurin VB. Fundamentals of the general theory of water sports locomotion. Teoriia i praktika fizicheskoj kul'tury. 1985;8: 45-50. (In Russian)

7. Krylov AI, Vinogradov EO. Quantitative estimation of headway movement of the swimmer. Kul'tura fizicheskaia $i$ zdorov'e, 2018;2:66-72. (In Russian)

8. Krylov AI, Butov AA, Vinogradov EO. Correction of swimming techniques using dynamic characteristics of the stroke. Uchenye zapiski universiteta imeni P.F. Lesgafta, 2016;2:132-140. (In Russian)

9. Krylov AI, Butov AA, Vent D. Natatometer. Realtime intra-cycle velocity data for swimming stroke correction. Uchenye zapiski universiteta imeni P.F. Lesgafta,2014;1:12-20. (In Russian) https://doi.org/10.5930/issn.1994-4683.2014.07.113.p109113

10.Platonov VN. Swimming. Kiev: Olympic literature; 2000. (In Russian)

11.Arishin AV, Pogrebnoj AI. Cognate development of physical and technical preparedness of highly qualified swimmers in
12.Mosunov DF, Mosunova MD, Grigor'eva DV, Pavliukevich $\mathrm{KN}$, Iarygina MA. Methodology of realization of cyclic perfection potential in paralympic swimming technique. Uchenye zapiski universiteta imeni P.F. Lesgafta, 2018;4:158-164. (In Russian)

13.SeifertL, Komar J, CrettenandF, Millet GP, DadashiF,Aminian $\mathrm{K}$. Inter-limb coordination and energy cost in swimming. Journal of Science and Medicine in Sport. 2017; 17: 4- 10. https://doi.org/10.1016/j.jsams.2013.07.003

14.Krylov AI, Butov AA, Vinogradov EO. Metrological analysis of method "natatometry" at the study of inside cycle speed of swimming. Uchenye zapiski universiteta imeni P.F. Lesgafta, 2018;2:156-162. (In Russian)

15. Barbosa TM, Bragada JA, Reis VM, Marinho DA, Carvalho C, Silva JA. Energetics and biomechanics as determining factors of swimming performance: updating the state of the art. Journal of Science and Medicine in Sport. 2010;13:20- 29. https://doi.org/10.1016/j.jsams.2009.01.003

16.Mosunov DF, Mosunova MD, Nazarenko IuA, Kleshnev IV, Pavliukevich KN, Makeev AS. Technology of realization the reserve of sports and technical perfection in the training of paralympic swimmer. Uchenye zapiski universiteta imeni P.F. Lesgafta, 2017;8:150-159. (In Russian)

17.Noskov MS, Gorelov AA, Tretyakov AA. To the question of individualization of the training process swimmers, sprinters highest qualification. Ekonomicheskie $i$ gumanitarnye issledovaniia regionov. 2015; 1. (In Russian)

18. Martens J, Daly D, Deschamps K, Staes F, Fernandes RJ. Inter-individual variability and pattern recognition of surface electromyography in front crawl swimming. Journal of Electromyography and Kinesiology. 2016;31:25- 31. https://doi.org/10.1016/j.jelekin.2016.08.016

19.Psycharakis SG, Sanders RH, Psycharakis SG. Validity of the use of a fixed point for intracycle velocity calculations in swimming. Journal of Science and Medicine in Sport. 2009;12:34- 40. https://doi.org/10.1016/j.jsams.2007.11.008 


\section{Information about the authors:}

Krylov A.I.; http://orcid.org/0000-0002-4805-7976; krylov56@gmail.com; St. Petersburg National State University of Physical Culture, Sport and Health P.F. Lesgaft; Dekabristov st., 35, St. Petersburg, Russia.

Gorelov A.A.; http://orcid.org/0000-0002-1067-1110; alegor5@mail.ru; St. Petersburg University of the Ministry of Internal Affairs of Russia; Pilot Pilutov st., 1, St. Petersburg, Russia.

Tretyakov A.A.; (Corresponding author); http://orcid.org/0000-0001-7498-6675; delphin87@inbox.ru; Belgorod law Institute of the Ministry of internal affairs of Russia named after I.D. Putilin; Gorky str., 71, Belgorod, Russia.

Cite this article as:

Krylov Al, Gorelov AA, Tretyakov AA. Integral indicators of the swimming techniques effectiveness of highly qualified crawlstroke swimmers. Pedagogics, psychology, medical-biological problems of physical training and sports, 2019;23(4):169-175. https://doi.org/10.15561/18189172.2019.0402

This is an Open Access article distributed under the terms of the Creative Commons Attribution License, which permits unrestricted use, distribution, and reproduction in any medium, provided the original work is properly cited (http://creativecommons.org/licenses/by/4.0/deed.en).

Received: 08.05.2019

Accepted: 12.06.2019; Published: 29.08.2019 